\title{
Gp120/CD4 Blocking Antibodies Are Frequently Elicited in ART-Naïve Chronically HIV-1 Infected Individuals
}

\author{
Jorge Carrillo ${ }^{1 \mathrm{*}}$, Luis Manuel Molinos-Albert ${ }^{1,2}$, Maria Luisa Rodríguez de la \\ Concepción ${ }^{1}$, Silvia Marfil ${ }^{1}$, Elisabet García ${ }^{1}$, Ronald Derking ${ }^{3}$, Rogier W. Sanders ${ }^{3,4}$, \\ Bonaventura Clotet ${ }^{1,5,6,7}$, Julià Blanco ${ }^{1,2,5,6}$
}

1 Institut de Recerca de la SIDA-IrsiCaixa-HIVACAT, Badalona, Barcelona, Spain, 2 Institut de Recerca en Ciències de la Salut Germans Trias i Pujol (IGTP), Hospital Germans Trias i Pujol, Badalona, Barcelona, Spain, 3 Department of Medical Microbiology, Academic Medical Center, University of Amsterdam, Amsterdam, The Netherlands, 4 Department of Microbiology and Immunology, Weill Medical College of Cornell University, New York, New York, United States of America, 5 Universitat Autònoma de Barcelona, Cerdanyola del Vallés, Barcelona, Spain, 6 Universitat de Vic-Central de Catalunya, UVIC-UCC, Vic, Barcelona, Spain, 7 Fundació Lluita contra la SIDA, Badalona, Barcelona, Spain

a Current address: Centre de Recerca en Salut Internacional de Barcelona (CRESIB), Barcelona, Spain * jcarrillo@ irsicaixa.es

\section{G open Access}

Citation: Carrillo J, Molinos-Albert LM, de la Concepción MLR, Marfil S, García E, Derking R, et al. (2015) Gp120/CD4 Blocking Antibodies Are Frequently Elicited in ART-Naïve Chronically HIV-1 Infected Individuals. PLOS ONE 10(3): e0120648. doi:10.1371/journal.pone.0120648

Academic Editor: Paul Zhou, Pasteur Institute of Shanghai, Chinese Academy of Science, CHINA

Received: October 10, 2014

Accepted: January 25, 2015

Published: March 24, 2015

Copyright: @ 2015 Carrillo et al. This is an open access article distributed under the terms of the Creative Commons Attribution License, which permits unrestricted use, distribution, and reproduction in any medium, provided the original author and source are credited.

Data Availability Statement: All relevant data are within the paper and its Supporting Information files.

Funding: This work was supported by the HIVACAT Program and the Spanish AIDS network 'Red Temática Cooperativa de Investigación en SIDA (RD06/0006)' and Fondo de Investigaciones Sanitarias (grant number PI1102089). J.B. is a researcher from Fundació Institut de Recerca en Ciències de la Salut Germans Trias i Pujol supported by the ISCIII and the Health Department of the Catalan Government (Generalitat de Catalunya). J.C. is supported by a 'Sara Borrell' grant from the

\section{Abstract}

Antibodies with the ability to block the interaction of HIV-1 envelope glycoprotein (Env) gp120 with CD4, including those overlapping the CD4 binding site (CD4bs antibodies), can protect from infection by HIV-1, and their elicitation may be an interesting goal for any vaccination strategy. To identify gp120/CD4 blocking antibodies in plasma samples from HIV-1 infected individuals we have developed a competitive flow cytometry-based functional assay. In a cohort of treatment-naïve chronically infected patients, we showed that gp120/ CD4 blocking antibodies were frequently elicited (detected in $97 \%$ plasma samples) and correlated with binding to trimeric HIV-1 envelope glycoproteins. However, no correlation was observed between functional CD4 binding blockade data and titer of CD4bs antibodies determined by ELISA using resurfaced gp120 proteins. Consistently, plasma samples lacking CD4bs antibodies were able to block the interaction between gp120 and its receptor, indicating that antibodies recognizing other epitopes, such as PGT126 and PG16, can also play the same role. Antibodies blocking CD4 binding increased over time and correlated positively with the capacity of plasma samples to neutralize the laboratory-adapted NL4.3 and $\mathrm{BaL}$ virus isolates, suggesting their potential contribution to the neutralizing workforce of plasma in vivo. Determining whether this response can be boosted to achieve broadly neutralizing antibodies may provide valuable information for the design of new strategies aimed to improve the anti-HIV-1 humoral response and to develop a successful HIV1 vaccine. 
Spanish Health Institute 'ISCIII'. LMM-A. is supported by a predoctoral grant from Generalitat de Catalunya and European Social Fund. The funders had no role in study design, data collection and analysis, decision to publish, or preparation of the manuscript.

Competing Interests: $\mathrm{BC}$ and JB have received fees for consultancy from BMS, GILEAD, ViiV or Jansen outside the submitted work. The huCD $4 / \mathrm{mlg}$ protein is protected by the WO 2014/037490 patent. JC and JB are inventors of the patent WO2014/037490: "Methods for Identifying HIV-1 Neutralizing Antibodies", in which the assay used for the identification of gp120/CD4 blocking antibodies is described. This does not alter the authors' adherence to Plos One policies on sharing data and materials.

\section{Introduction}

During the course of an infection process the infected organism develops a broad humoral response, which in conjunction with the innate and T-cell response, control the infection and preserve the integrity of the organism. In the setting of the HIV-1 infection, this humoral response can be detected early during the infection but it is known to be ineffective to control viral replication [1], mainly because the antibodies produced by most patients are nonneutralizing, i. e. recognize viral epitopes that fail to interfere with the replicative cycle of the virus. Although in some patients, antibodies with the capacity to neutralize the autologous virus can be identified after few months of infection, several years are required to develop broadly neutralizing antibodies (bNAbs) [2-4]. To date, many broadly neutralizing antibodies have been identified and all of them recognize epitopes within the HIV-1 envelope glycoprotein (Env) with an important role in viral fitness [2]. These antibodies include CD4 binding site (CD4bs) antibodies (IgGb12 [5] and VRC01 [6]), anti-CD4 induced-epitope antibodies (CD4i antibodies) (X5 [7]), anti-gp41 antibodies (2F5 [8], 4E10 [9] and 10E8 [10]), anti-carbohydrates (2G12 [11]), anti-glycosylated quaternary epitopes (PG9 and PG16) [12] and anti-core antibodies [13]. Among them, antibodies with the ability to block the interaction of gp 120/CD4 such as CD4bs antibodies should be highlighted for several reasons: 1) they recognize a conserved region of gp120,2) they can neutralize a broad number of viral isolates and 3) they can prevent or control the infection in animal models of HIV-1 infection [14,15]. Therefore, the elicitation of this sort of bNAbs is an interesting goal for any vaccination strategy. However, one of the major handicaps in the study of these antibodies is their identification. Broadly neutralizing antibodies in general and CD4bs antibodies in particular, recognize conformational epitopes, which are difficult to mimic in vitro. To date, several strategies have been followed to study the CD4bs antibodies, these include the use of recombinant proteins and mutant variants which are differentially recognized by these antibodies $[6,16,17]$. Also our team has recently developed a cellto-cell viral transfer assay, which enables the identification of gp120/CD4 blocking antibodies in plasma samples [18]. This assay is based on the viral entry process, which is blocked at early stages in the presence of antibodies that block the gp120/CD4 interaction, like CD4bs antibodies. Furthermore, this work showed a positive correlation between the presence of gp120/CD4 blocking antibodies and the neutralizing capacity of the plasma, being the samples showing this specificity the ones with broader and more powerful neutralizing responses [18]. Interestingly, using a set of recombinant proteins, Lynch et al. have shown that more than $80 \%$ of HIV-1 infected patients can develop CD4bs antibodies, indicating that this reactivity might be more frequent than it had been previously described [17]. However, in the latter study a clear correlation between the presence of these antibodies and the neutralizing capacity of the plasma samples was not observed.

To improve the identification of gp120/CD4 blocking antibodies we have developed a competitive flow cytometry-based assay, which enables the detection of these antibodies in plasma or serum samples. This assay is based on the recognition of the Env glycoprotein, expressed on the surface of HIV-1 persistently infected cell lines, by the use of a newly designed huCD4/ mouse-IgG (huCD4mIgG) fusion protein. The results showed that the presence of gp120/CD4 blocking antibodies is more represented in HIV-1-chronically infected patients than expected. The levels of these antibodies did not show correlation with the titer of CD4bs antibodies, probably because they may target CD4bs but also other unrelated epitopes. Finally, these antibodies correlate with the neutralizing capacity of plasma samples assayed using laboratoryadapted virus isolates. 


\section{Methods}

\section{Samples}

A total of 10 uninfected healthy donors and 36 untreated HIV-1 infected individuals with viral load (VL) $>50$ copies/mL and with at least two plasma samples separated by one year were selected retrospectively. Main patient's characteristics are described in Table 1 (mean \pm standard deviation). Since it was not possible to establish exactly the date of infection for each patient, time after diagnosis of HIV-1 infection was used as an estimation of infection length. Plasma was prepared by blood centrifugation for 10 minutes at 3000xg.

The data presented here are part of the observational study "ANALYSIS OF HUMORAL RESPONSES IN UNTREATED HIV+ PATIENTS”. The study was carried out at the Hospital Universitari Germans Trias i Pujol (Badalona, Spain) and was approved by the Ethics Committee of the Hospital Universitari Germans Trias i Pujol (PI081306). All participants provided written informed consent to participate in the study.

\section{Production of the huCD4mlgG recombinant protein}

The D1-D2 N-terminal domains of the human CD4 molecule were amplified by standard RT-PCR using the SuperScript III One-Step RT-PCR System with Platinum Taq DNA polimerase (Invitrogen) and the following primers:

CD4 L sense: 5' -CACCATGAACCGGGGAGTCCCTTTTAG-3' and

\section{CD4L AS NheI: 5' -TATTAGCTAGCACCACGATGTCTATTTTG-3'.}

RNA extracted from human peripheral blood mononuclear cells (PBMC) was used as template. The pcDNA3.1huCD4 plasmid was generated after cloning of the CD4 amplimer using the pcDNA3.1 Directional V5-His-TOPO kit and following the manufacturer's instructions.

The hinge/CH2/CH3 containing-Fc region of murine IgG1 was amplified using the primers:

mIgG1-S: 5'-GAATAGAGCTGGTGGGCTAGCTGTGCCCAGGGATTGTGGT-3' and mIgG1-AS: 5'-TTATTCTCGAGTCATTTACCAGGAGAGTGGG-3' .

As template, RNA extracted from the NS1 (ATCC) murine cell line was used.

The amplimer was purified, digested with the FastDigest NheI and XhoI restriction enzymes (Fermentas) and ligated into the pcDNA3.1huCD4 (previously linearized with the same restrictions enzymes) using T4 DNA ligase (Fermentas). The DNA-construct integrity was confirmed by sequencing using the BigDye Terminator v3.1 Cycle Sequencing Kit (Applied Biosystems). To produce the recombinant fusion protein, 293 cells (NIH AIDS reagent program) were transfected with the pcDNA3.1huCD4mIgG1 plasmid using Calphos transfection kit (Clontech) following the manufacturer's instructions. After 48 hours, the supernatant was

Table 1. Characteristic of ART-naive HIV-1 infected patients.

\begin{tabular}{|c|c|}
\hline $\mathbf{n}$ & 36 \\
\hline$\%$ CD4 & $28.3 \pm 7.1$ \\
\hline CD4/ $\mu \mathrm{L}$ & $586 \pm 216$ \\
\hline$\%$ CD8 & $52.2 \pm 10$ \\
\hline 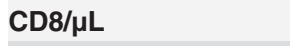 & $1079 \pm 416$ \\
\hline VL (copies/mL) & $51127 \pm 183286$ \\
\hline Days after diagnosis & $1803 \pm 1933$ \\
\hline
\end{tabular}

doi:10.1371/journal.pone.0120648.t001 
collected, clarified by filtration through a $0.45 \mu \mathrm{m}$ filter (Millipore) and stored at $-20^{\circ} \mathrm{C}$ until use.

\section{Quantification and titration of supernatants containing the huCD4mlgG recombinant protein}

$\mathrm{HuCD} 4 \mathrm{mIgG}$ recombinant protein was quantified by an in house ELISA using goat antimouse IgG(Fc specific) (Jackson-Immunoresearch) as capture antibody, Rat anti-mouse IgG1 (Biolegend) as secondary antibody and HRP-Goat anti-Rat IgG as detection antibody (Jackson-Immunoresearch). Serial dilutions of purified IgG1-monoclonal antibody clone SK3 (BD Biosciences) was used as standard. Using this approach the concentration of the recombinant protein was equivalent to $11 \mu \mathrm{g} / \mathrm{mL}$ of mouse IgG1. All concentration values of huCD4mIgG fusion protein are given in $\mu \mathrm{g} / \mathrm{mL}$ of equivalent mouse IgG1.

HIV-1 NL4.3 chronically-infected MOLT cells [19] were incubated with serial dilutions of the huCD4mIgG-containing supernatant for 30 minutes at room temperature. After washing with PBS, the huCD4mIgG fusion protein bound to gp120 on the infected-cell surface was detected by flow cytometry using a Fc-specific DyLight 649-F(ab)2 Goat anti-mouse IgG (Jackson-Immunoresearch).

\section{Detection of gp120/CD4 blocking antibodies using a competitive- cytometric assay}

HIV-1 NL4.3 and BaL chronically-infected MOLT cells [19] were pre-incubated at room temperature for 25 minutes with serial dilutions of plasma samples or monoclonal antibodies VRC01, VRC03 [6], PG9, PG16 [12], PGT 126 [20] (NIH AIDS Reagent Program), IgGb12, 2G12, 2F5, 447-52D (Polymun), or the polyclonal antibody Goat anti-gp120 (Abcam). Then, the huCD4mIgG-containing supernatant at $0.22 \mu \mathrm{g} / \mathrm{mL}$ was added and the incubation extended for 30 minutes at room temperature. After two washes with PBS, the Fc-specific secondary antibody DyLight 649-F(ab)2 Goat anti-mouse IgG (Jackson-Immunoresearch) was added and incubated again for 15 minutes at room temperature. The cell samples were washed, fixed in $1 \%$ formaldehyde (Sigma-Aldrich) and analyzed by flow cytometry.

\section{Quantification of anti-Env antibodies in plasma samples by flow cytometry}

Anti-Env antibodies were quantified as previously described [21]. Briefly, MOLT-NL43, MOLT-BaL and uninfected MOLT cell line were stained with plasma samples at 1/100 dilution, in PBS $+10 \%$ fetal bovine serum (Life technologies), for 30 minutes at room temperature. After washing with PBS, bound antibodies were detected with a PE-Goat anti-human IgG secondary antibody (Jackson-Immunoresearch). Specific signal was determined as the ratio between the Mean Fluorescent Intensity (MFI) obtained for each infected cell line (NL43 or BaL) and the uninfected one.

\section{Quantification of anti-Env antibodies in plasma samples by ELISA}

Titer of IgG antibodies binding to trimeric Env glycoprotein was determined by ELISA using a D7324-tagged version of the recently described soluble HIV-1 Env trimer BG505 SOSIP.664 gp140 and following the methods described by Sanders et al [22]. Briefly, Maxisorb Elisa plates (Nunc) were coating with D7324 antibody $(10 \mu \mathrm{g} / \mathrm{mL})$ and blocked using TBS+10\%FBS for two hours at room temperature. After washing, the BG505 SOSIP.664 protein was added at $1 \mathrm{ng} / \mathrm{ml}$ in blocking buffer and incubated overnight at $4^{\circ} \mathrm{C}$. Then, plates were washed and 
blocked again using TBS/2\% skimmed milk. After washing, plasma samples diluted 1/1000 in $\mathrm{TBS} / 5 \% \mathrm{FBS} / 2 \%$ skimmed milk were added and incubated for two hours at room temperature. HRP-Goat anti-human IgG (Fc specific) (Jackson-Immunoresearch) was used as secondary antibody. The reaction was revealed using 3, 3',5, 5' -Tetramethylbenzidine (Sigma-Aldrich) and stopped using $2 \mathrm{M}_{\text {of }} \mathrm{H}_{2} \mathrm{SO}_{4}$. $2 \mathrm{G} 12$ antibody was used as standard and the results are showed as arbitrary units. The IgGb12 antibody, which does not bind to the BG505 SOSIP.664, was used as negative control. Plasma samples were assayed in parallel in D7324 antibody coated and antigen free wells to evaluate background. The signal obtained in these wells was subtracted to the signal obtained with antigen.

\section{Determination of CD4bs antibodies by ELISA}

The presence of CD4bs antibodies was quantified by ELISA using the recombinant protein RSC3 and RSC3 3371 I (RSC3 $\Delta$ ) (NIH AIDS Reagent Program). RSC3 exposes the CD4bs of gp120, and RSC3 $\Delta$ is a truncated protein with reduced binding of CD4bs antibodies [6,17]. Briefly, $50 \mathrm{ng} /$ well of protein in PBS was bound to 96-well Maxisorb plates (NUNC) overnight at $4^{\circ} \mathrm{C}$. After washing and blocking the wells with PBS plus $10 \%$ of FBS, plasma samples diluted in blocking buffer were added and incubated overnight at $4^{\circ} \mathrm{C} .2 \mathrm{G} 12$ monoclonal antibody was used as standard and IgGb12, which recognized RSC 3 but not RSC $3 \Delta$, was used as control of CD4bs antibodies. Anti-CD4bs titer was calculated for each plasma sample as the titer of antiRSC3 antibodies minus titer of anti-RSC3 $\Delta$ antibodies (RSC3- RSC3 $\Delta$ ).

\section{Neutralization capacity of plasma samples}

Neutralization assays were as described [18]. Plasma samples, inactivated for 1 hour at $56^{\circ} \mathrm{C}$, were used to evaluate virus-specific neutralization activity, using the TZM-bl neutralization assay [23]. Briefly, three-fold serial dilutions of plasmas were incubated in 96-well plates in duplicates for 1 hour with 200 TCID50 of two laboratory-adapted viral strains (HIV-1 NL4-3 and $\mathrm{BaL}$ ) and two primary isolates (AC10 and SVPB16). $10^{5} \mathrm{TZM}$-bl cells were added per well and incubated for 48 hours at $37^{\circ} \mathrm{C}$ and $5 \% \mathrm{CO}_{2}$. Luciferase activity was determined using the BriteLite plus (PerkinElmer) and the luminescence was then quantified in a luminometer (Fluoroskan Ascent FL, Labsystem). The relative light units (RLU) obtained were used to calculate the percentage of neutralization using the formula: \% Neutralization $=[1-(\mathrm{R}-\mathrm{Rcc} / \mathrm{Rvc})] \times$ 100 ; where $\mathrm{R}=\mathrm{RLU}$ of the tested plasma, $\mathrm{Rcc}=\mathrm{RLU}$ of cell alone and $\mathrm{Rvc}=\mathrm{RLU}$ of virus alone. The dose inducing $50 \%$ of total inhibitory capacity (IC50) was calculated and results are shown as reciprocal dilution.

\section{Statistic analysis}

Data were analyzed using GraphPad Prism and R (v.3.1.2) softwares. Fisher's exact test, MannWhitney U test, Paired t-test and Spearman's correlation were applied when required.

\section{Results}

\section{High prevalence of gp120/CD4 blocking antibodies in ART-naïve HIV-1 chronically infected patients}

Antibodies showing the capacity to block the interaction between gp120 and the CD4 receptor expressed on the surface of CD4+ T cells may be considered as putative neutralizing antibodies. To determine the presence of this kind of antibodies in our cohort of HIV-1 infected patients, we developed a flow cytometry-based competitive assay using a human-CD4/mouse-IgG fusion protein and two HIV-1-chronically infected MOLT cell lines (BaL and NL4.3). HIV-1 Env 
can be detected on the surface of these cells by anti-gp120 antibodies that recognize conformational-restricted trimer-specific (PG9, PG16) and non-conformational restricted epitopes, such as IgG2G12, PGT126 or CD4bs antibodies (IgGb12, VRC03 and VRC01), with similar results (S1 Fig.). Moreover, antibodies targeting epitopes that are poorly exposed (such as MPER) or only exposed after gp120/CD4 interaction (CD4-induced) showed low or negligible signal, respectively (S1 Fig.). Despite differences among the former staining due to different affinity/ avidity of antibodies, the results strongly suggested that the chronically-infected MOLT cell lines mainly expressed a functional-native Env glycoprotein on their surface (S1 Fig.). Similar results were obtained with MOLT-BaL (data not shown).

MOLT infected cell lines could also be stained with the huCD4mIgG recombinant protein (Fig. $1 \mathrm{~A}$ and $\mathrm{B}$ ) and the addition of the IgGb12 antibody, which recognizes the CD4 binding site in gp120, blocked the interaction between the huCD4mIgG and gp120 in a concentration dependent way, indicating that this inhibition could be used as a measure of the presence of antibodies directed against the CD4 binding site of gp120 (Fig. 1C). Following this approach, we determined the prevalence of gp120/CD4 blocking antibodies in plasma samples from ARTnaïve HIV-1 infected individuals. When BaL chronically-infected MOLT cell lines were used, gp120/CD4 blocking antibodies were detected in 34 out of 35 of samples tested (97\%, Fig. 2A), and showed a good correlation with the gp120/CD4 blocking antibodies detected using MOLT-NL43 ( $\mathrm{r}=0.7, \mathrm{p}<0.0001)$ (Fig. 2B).

When plasma samples obtained from the same patients after one year of untreated infection were analyzed, statistically significant differences were found between both time points, supporting that the presence of these antibodies increased over time $(\mathrm{p}=0.004)$ (Fig. 2C). According to that, the presence of this kind of antibodies correlated with the time after diagnosis (Fig. 2D) indicating that a prolonged exposure to the virus may drive the development of these antibodies. However, a poor correlation was found between the presence of gp120/CD4 blocking antibodies and the viral load $(\mathrm{r}=0.34, \mathrm{p}=0.047)$ (Fig. 2E), suggesting that viremia may not be a major factor for the development and or maintenance of these antibodies although a certain level of virus may be required.

\section{Detection of CD4bs antibodies in plasma samples by ELISA}

Given that CD4bs antibodies are able to block the interaction between gp120 and CD4, the presence of these antibodies was quantified by ELISA. Each plasma sample was assayed against a protein that exposes the CD4bs and can be recognized by CD4bs antibodies (RSC3); and a mutated version of this protein (RSC3 $\Delta$ ) which shows an impaired reactivity for most of CD4bs antibodies (Fig. 3A) [6,17]. Therefore, results obtained after subtracting anti-RSC3 $\Delta$ titers to anti-RSC 3 titers were used as an estimation of CD4bs antibodies. The results showed that 25 out of 36 analyzed patients (70\%) showed this sort of antibodies whereas none of the healthy uninfected controls were positive ( $\mathrm{p}<0.0001$, Fisher's exact test) (Fig. 3B). No correlation was observed between CD4bs antibodies titer, VL, CD4+ T cells count and time from diagnosis (data not shown). To determine the stability of the presence of these antibodies, we quantified the CD4bs antibodies in subsequent samples from the same patients in the absence of antiretroviral therapy (Fig. 3C). No differences were observed between both time points, suggesting that this kind of antibodies is generally maintained in chronic infection, at least for one year. Just one patient developed and other two become undetectable for the presence of CD4bs antibodies after one year, indicating some extent of variability among individuals. These results confirm that CD4bs antibodies are commonly generated in HIV-1 chronically infected patients and are in line with previously published data[17]. 
A

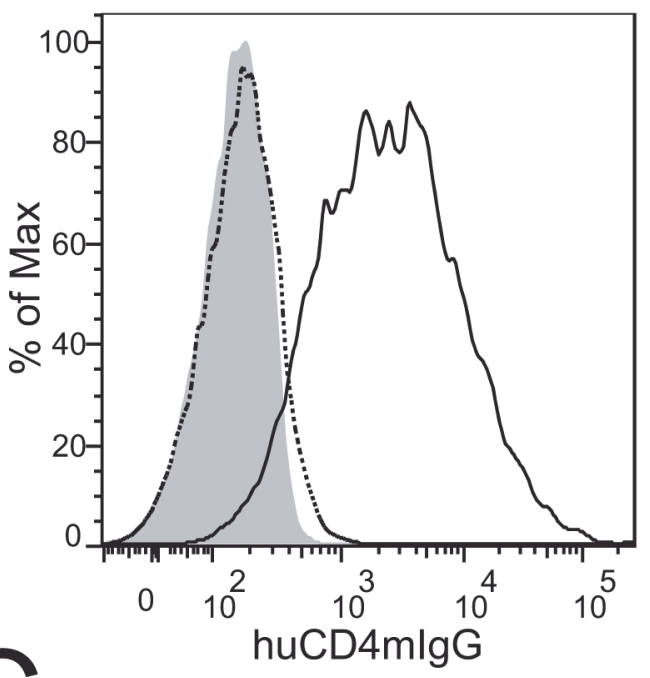

B

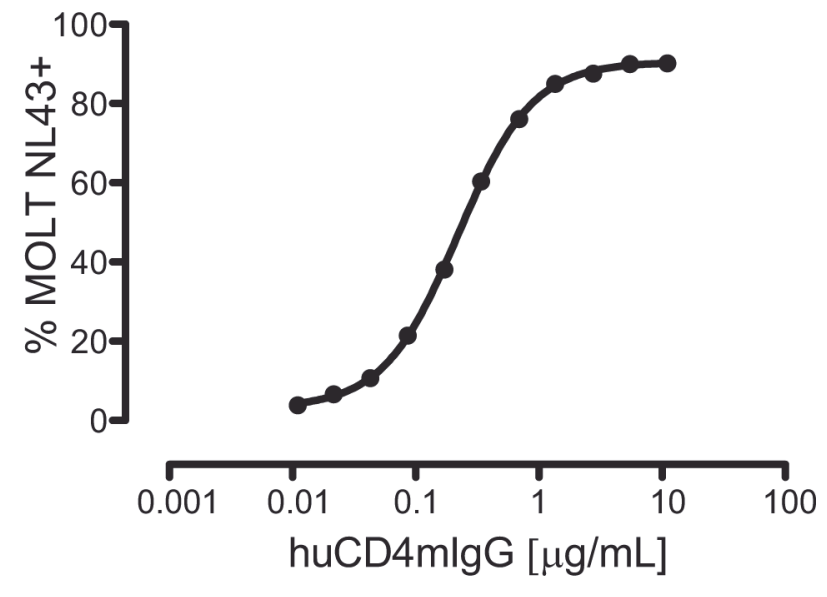

C

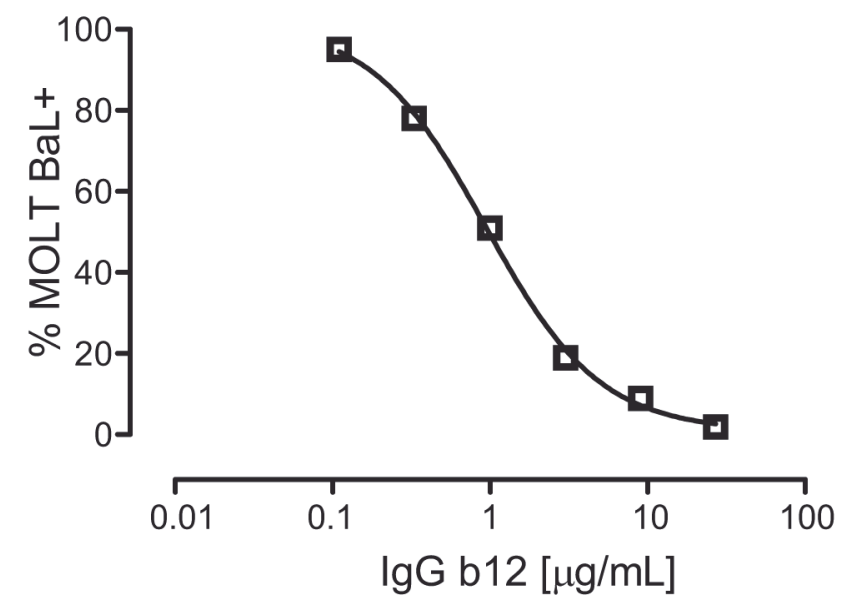

Fig 1. Identification of gp120/CD4 blocking antibodies. The presence of antibodies with the capacity to block the interaction between CD4 and gp120 was evaluated with a flow cytometry competitive assay using a chronically infected cell line and a huCD4mlgG recombinant protein. A) MOLT-NL4.3 (black line) and uninfected-MOLT cells (gray) were stained with huCD4mlgG $(10 \mu \mathrm{g} / \mathrm{mL})$ and a DyLight-649 Fab2 Goat anti-mouse lgG as secondary antibody. Dotted line shows the staining of MOLT-NL43 cell using a mouse lgG1 isotype control. B) MOLT-NL4.3 cells were stained with serial dilutions of huCD4mlgG. C) IgGb12 inhibition of the binding of huCD4mlgG to MOLT-NL4.3.

doi:10.1371/journal.pone.0120648.g001

\section{CD4bs antibodies are not correlated with gp120/CD4 blocking activity of plasma}

To determine whether CD4bs antibodies were linked to gp120/CD4 blocking activity, a correlation analysis was performed. The results did not show any correlation between both parameters $(r=0.2612, p=0.1296$, Fig. 4A). Some plasma samples with high titers of CD4bs antibodies showed a poor gp120/CD4 blocking activity suggesting that these antibodies may be nonneutralizing antibodies. On the other hand, a subset of samples (28\%, 10 out of 36$)$ did not apparently contain CD4bs antibodies but were able to prevent the binding of gp120 to its receptor, suggesting that CD4bs antibodies that are able to recognize equally both RSC3-based 

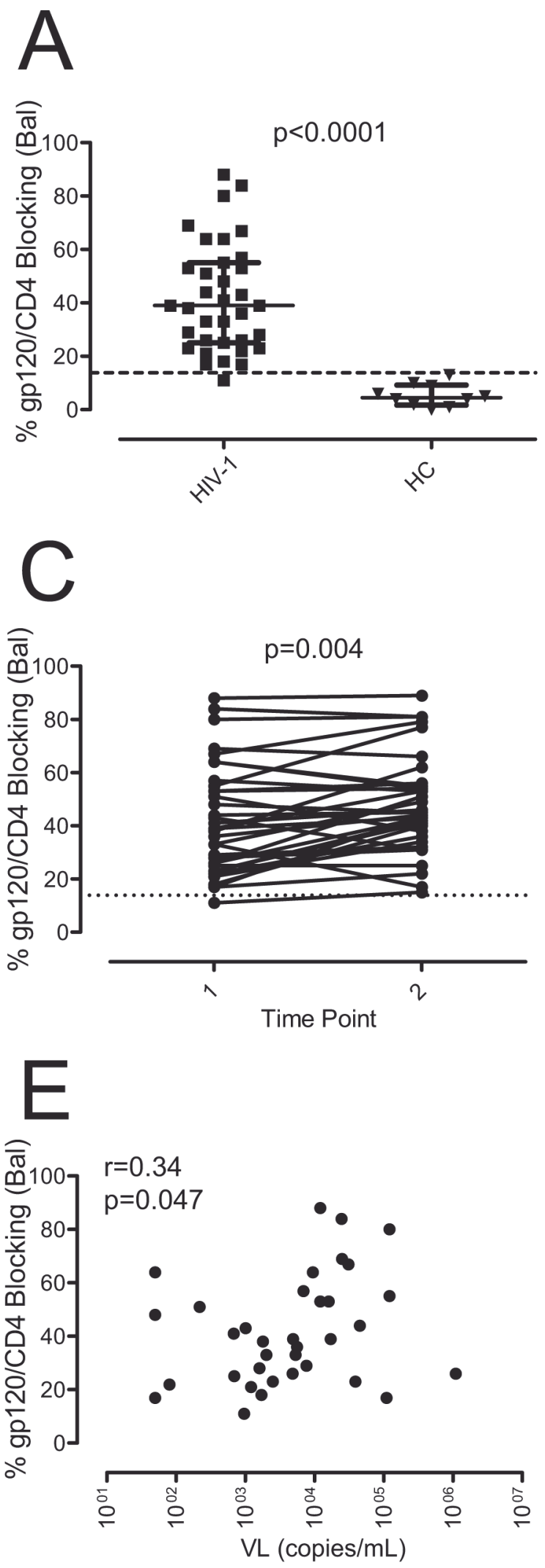
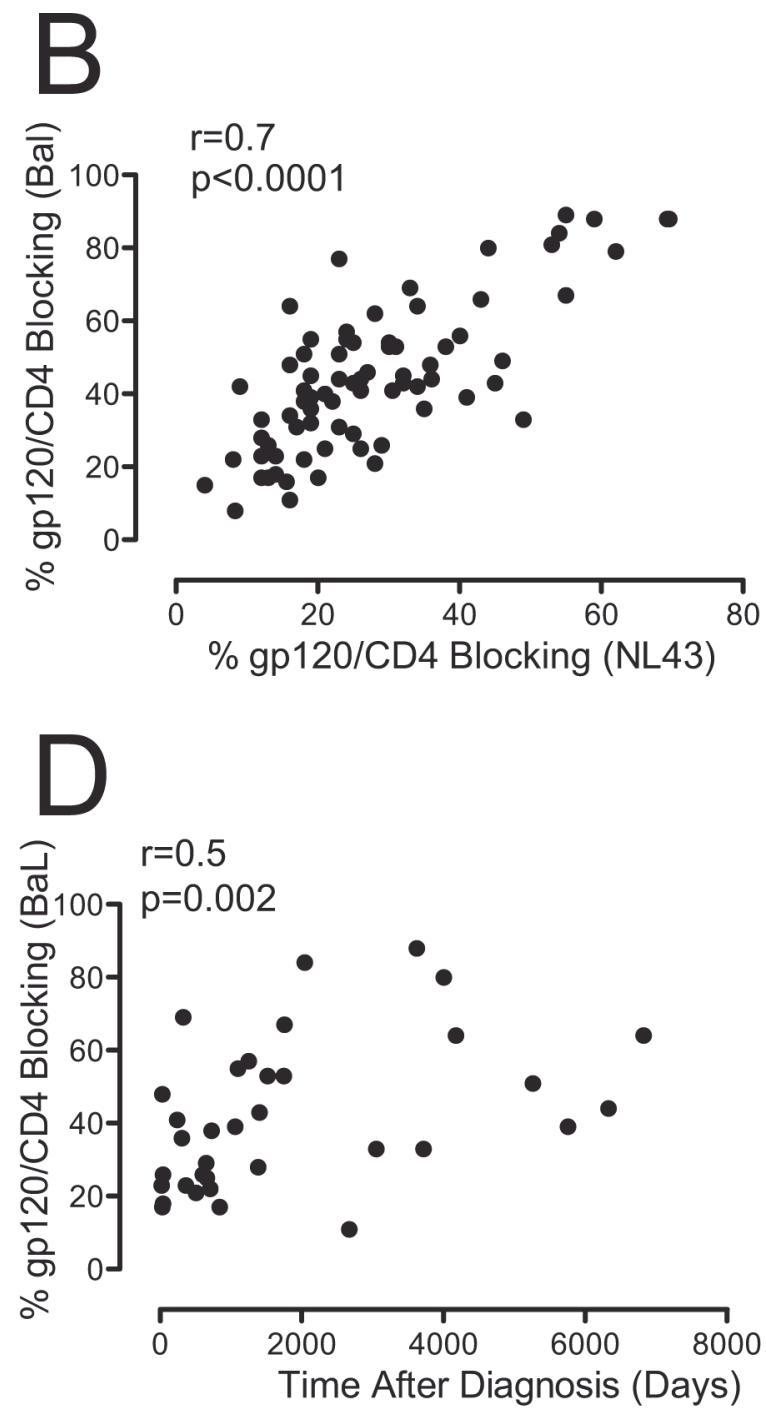

Fig 2. Gp120/CD4 blocking antibodies in ART-naive HIV-1 infected patients. A) The presence of gp120/CD4 blocking antibodies was determined in the plasma of 35 ART-naive HIV-1 infected patients (squares) and compared with 10 uninfected healthy control individuals (HC, triangles). The cytometric 
competitive assay was performed using the huCD4mlgG recombinant protein and the MOLT-BaL cell line. Differences were calculated using Mann-Whitney U test. Dotted line shows cut-off of positivity calculated as mean+2xSD of HC samples. B) Correlation between levels of gp120/CD4 blocking antibodies, using MOLT-BaL and MOLT-NL4.3 cells, was determined by Spearman's test. C) Gp120/CD4 blocking antibodies were determined in two plasma samples separated by one year from the same HIV-1 infected individuals (paired t-test). Dotted line shows cut-off of positivity as in panel A. D and E) Correlation analysis between the percentage of gp120/CD4 blocking activity of plasma samples and time after diagnosis or viral load (VL) was performed using Spearman's correlation.

doi:10.1371/journal.pone.0120648.g002

proteins or antibodies elicited against other region than CD4bs can also block the interaction of gp120 with its receptor. To evaluate these options, we quantified by ELISA the presence of antibodies that recognize the trimeric Env protein using the soluble trimer BG505 SOSIP.664 gp 140. 27 out of $31 \mathrm{HIV}-1+$ plasma samples analyzed were positive. The mean+2xSD of the signal obtained with plasma samples from uninfected healthy control was used as positivity cut-off (S2 Fig.). The titer of antibodies against the BG505 SOSIP.664 trimer showed a slight positive correlation with CD4bs antibodies (Spearman's $r=0.421, p=0.02$ ) (Fig. 4B). Similar results were obtained when the anti-Env humoral response was quantified by flow cytometry using MOLT-BaL cells (S2 Fig.), reinforcing that the Env glycoprotein expressed on the surface of these cells is mainly trimeric. Interestingly, most of plasma samples lacking CD4bs Abs were trimer-positive and showed some gp120/CD4 blocking activity; indicating that specificities other than the $\mathrm{CD} 4 \mathrm{bs}$ may mediate this action. In addition, when all three parameters (trimerspecific Abs, CD4bs Abs titer and gp120/CD4 blocking activity) were plotted altogether six plasma samples stood out that showed high BG505 SOSIP.664 trimer recognition (Fig. 4C). Of them, only two showed CD4bs antibodies and high potency to block the gp120 binding to $\mathrm{CD} 4$, suggesting that this plasma samples may contain high titers of neutralizing CD4bs antibodies. In contrast, 3 out of these 6 samples showing high recognition of the trimer and CD4bs $\mathrm{Ab}$ titer failed to efficiently block the binding of $\mathrm{CD} 4$ to trimeric Env, suggesting that $\mathrm{CD} 4 \mathrm{bs}$ antibodies present in these samples may be poorly neutralizing. A last sample lacked CD4bs antibodies but showed high anti-trimer titer and high gp120/CD4 blocking activity, suggesting that antibodies other than CD4bs are responsible of gp120/CD4 blocking activity of this sample.

\section{Plasma samples with gp120/CD4 blocking antibodies showed neutralizing capacity}

To test whether plasma samples containing gp120/CD4 blocking antibodies showed an improved neutralizing capacity, plasma samples were classified according to the presence of this sort of antibodies and CD4bs antibodies, and their neutralizing capacity assayed by a TZM-bl based neutralization assays. Similar results were obtained when plasma samples were classified according to BG505 SOSIP.664 binding and CD4bs Abs titer (Fig. 4B and data not shown). As a reference, four different HIV-1 viral isolates were used: two laboratory adapted (BaL and NL4.3) and two primary isolates (AC10 and SVPB16). As expected, most of the samples showed neutralization capacity (reciprocal IC50>60) against BaL (72\%, 26 out of 36 ) and NL4.3 (89\%, 32 out of 36), whereas only some of them could neutralize SVPB16 (39\%, 14 out of 36$)$ and $\mathrm{AC} 10(36 \%, 13$ out of 36$)$ but with poor potency as denote the low reciprocal IC50 of plasma dilution (Fig. 4D). To investigate the relative contribution of gp120/CD4 blocking antibodies to neutralizing capacity of plasma, a correlation analysis between both parameters was performed for each group (Fig. $4 \mathrm{E}$ and $\mathrm{F}$ ). The results showed a positive correlation between the presence of gp120/CD4 blocking antibodies and the neutralizing capacity of plasma samples for NL4.3 and BaL isolates, indicating that these antibodies may contribute to the neutralizing workforce of plasma, even though with low amplitude. Interestingly, some plasma samples showing a high gp120/CD4 blocking capacity showed a poor neutralizing activity, 

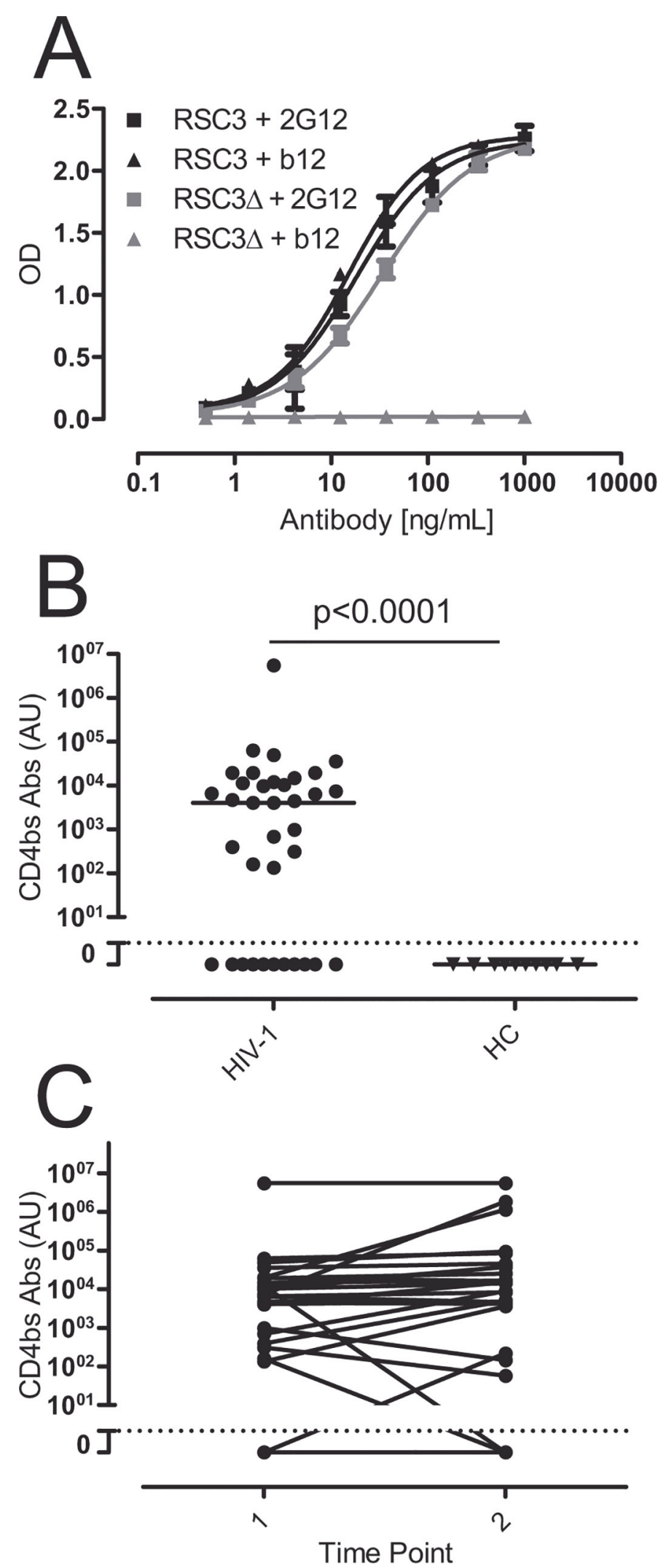
Fig 3. Detection of CD4bs antibodies by ELISA. The presence of CD4bs antibodies in ART-naive HIV-1 infected patients (HIV-1) was evaluated by ELISA using the RSC3 and the RSC3 3371 I (RSC $\Delta \Delta$ ) recombinant proteins. A) Standard curve was calculated using the $2 \mathrm{G} 12$ antibody, which recognized both recombinant proteins. The antibody IgGb12, which recognized RSC3 but not RSC3A, was used as control. B) The titer of CD4bs antibodies was calculated as RSC3-RSC3 $\Delta$. Differences with uninfected healthy donor controls $(\mathrm{HC})$ were evaluated by Fisher's exact test. Dotted line show cut-off of positivity calculated as mean $+2 \mathrm{xSD}$ of $\mathrm{HC}$ samples. C) Levels of CD4bs antibodies were determined in two plasma samples separated by one year from the same HIV-1 infected individuals. Dotted line indicated the cut-off as in panel B. No difference between both time points was observed (paired t-test).

doi:10.1371/journal.pone.0120648.g003

suggesting that other factors, such as sequence variation of target epitopes, might modulate the inhibition activity of these antibodies.

\section{Gp120/CD4 blocking activity is mediated by anti-gp120 defined specificities}

In order to determine whether the gp120/CD4 blocking activity of plasma samples may be mediated by a polyclonal Env-specific humoral response, a correlation analysis between the gp120/CD4 blocking activity and anti-Env IgG antibodies was performed. Anti-Env IgG was determined by flow cytometry using MOLT-BaL or MOLT-NL4.3 (not shown) and MOLT uninfected cells, and the specific signal expressed as MFI ratio. In addition, anti-Env IgG were also evaluated by ELISA using the soluble trimer BG505 SOSIP.664. The results showed a positive but modest correlation between the gp120/CD4 blocking capacity of plasma and anti-Env response suggesting again that the blocking activity would be mediated by defined antibodies and not simply by an Env-specific polyclonal response (Fig. 5A and 5B). To better characterize the specificity of the gp120/CD4 inhibition, several well-characterized antibodies were assayed in the same conditions (Fig. 5C). In addition to IgGb12, other antibodies directed against the CD4 binding site in gp120, such as VRC01, showed blocking activity. The PGT126 antibody, which recognize an epitope dependent on V3 loop and glycosylation at position 332/301 within gp120 [20], and PG9/PG16, which recognize a glycosylated-quaternary epitope strongly dependent on the native trimeric structure of the Env protein [12], also showed blocking capacity that was lower than IgGb12 or VRC01 antibodies. However, neutralizing antibodies such as 2G12, 447-52D, 2F5 and 4E10; and non-neutralizing polyclonal antibodies directed against other epitopes in gp120, failed to block the interaction between gp120 expressed on the surface of the cells and the huCD $4 \mathrm{mIgG}$ recombinant protein. These results suggested that the gp120/ CD4 blocking antibodies detected in plasma from HIV-1 infected patients may be the result of some defined specificities, mainly CD4bs antibodies but also other antibodies such as PGT126, PG16 or PG9-like, and cannot be explained by a polyclonal humoral response against HIV-1.

\section{Discussion}

Despite it is widely accepted that the humoral immune response is not able to clear HIV-1 infection once started, some patients develop broadly neutralizing antibodies whose efficacy protecting against the infection has been widely reported in defined settings. It has been shown that this kind of antibodies can protect against new infection in non-human primate models [24-28] and help to control viremia in chronically infected patients [29]. These observations make the development of this kind of antibodies one of the major goals of any vaccination strategy [2].

Although our knowledge on neutralizing antibodies has improved during the last years with the identification of new specificities, many questions still remain unresolved such as why they are only elicited in a restricted set of patients or why they take so much time to appear. One 

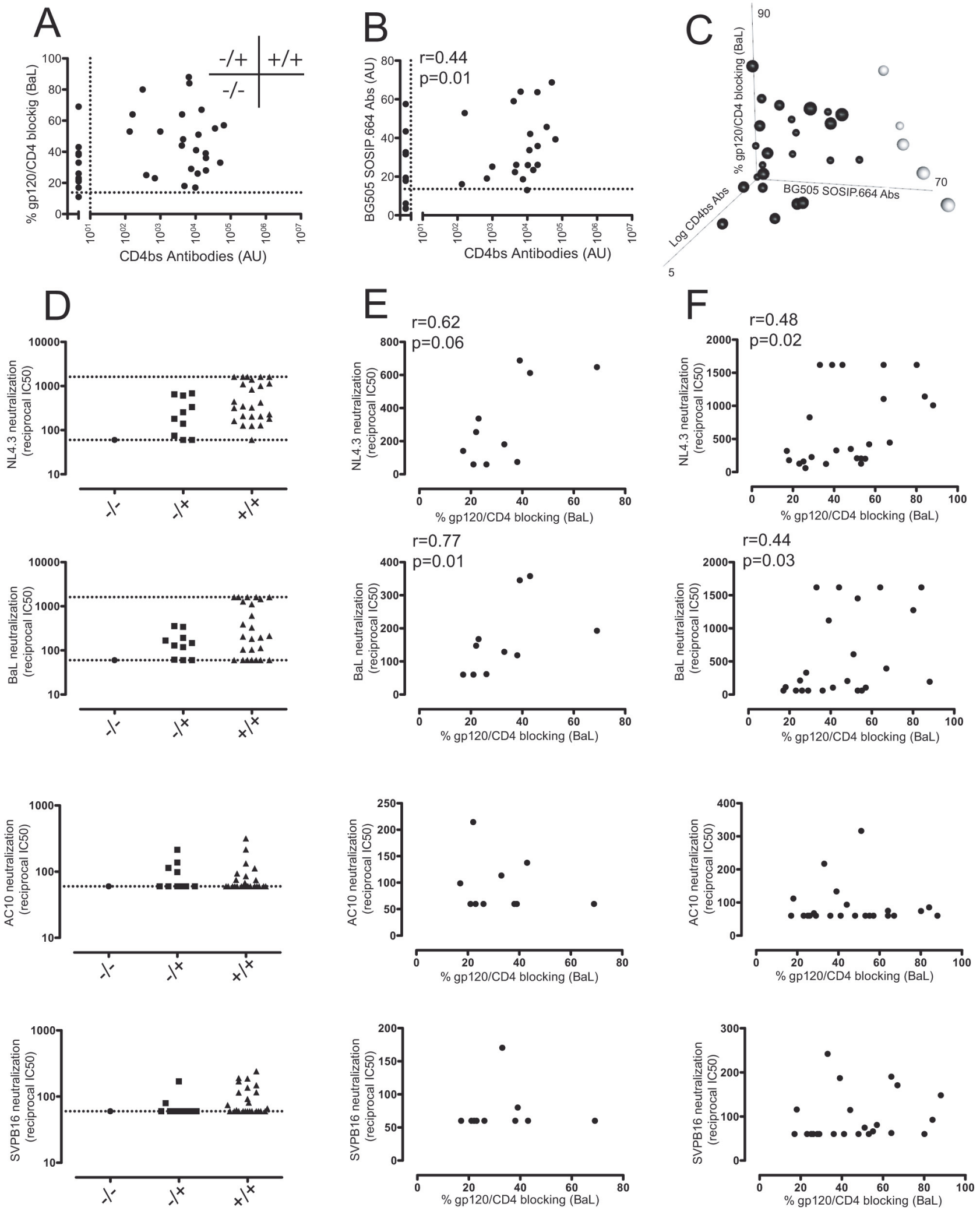

Fig 4. Correlation of gp120/CD4 blocking antibodies and CD4bs antibodies with the neutralizing capacity of plasma samples. A) Gp120/CD4 blocking activity showed no correlation with the titer of CD4bs antibodies (Spearman's correlation). Dotted line indicated the positivity cut-off of each 
parameter. Three groups of plasma samples were defined using cut-off values: plasma samples showing CD4bs and gp120/CD4 blocking antibodies (+/+), samples with detectable gp120/CD4 blocking Abs but undetectable CD4bs Abs (-/+) and plasma samples lacking both specificities (-/-).B) Titer of BG505 SOSIP.664 reactive antibodies showed a weak positive correlation with CD4bs Abs. Dotted line indicated the positivity cut-off of each parameter. Note that most of plasma samples negative for CD4bs Abs showed Env-reactive antibodies. C) gp120/CD4 blocking activity was plotted against the titer of BG505 SOSIP.664 binding antibodies and titer of CD4bs antibodies. Six plasma samples showing high reactivity against the trimer and variable levels of CD4bs antibodies and gp120/CD4 blocking activity are outlined (clear symbols). D) Neutralizing capacity of plasma samples were assayed using four viral isolates: NL4.3, BaL, AC10 and SVPB16. Samples were classified according to panel A. Dotted lines indicate upper and lower dilutions of plasma samples assayed. E) Samples lacking CD4bs Abs but showing gp120/CD4 blocking Abs (-/+) and F) samples showing both specificities (+/+) were analysed for correlation between gp120/CD4 blocking activity and neutralizing capacity (reciprocal IC50) using the viral isolates described in D. Spearman's correlation analysis was performed ( $r$ and $p$ values are only shown for significant correlations).

doi:10.1371/journal.pone.0120648.g004

intrinsic characteristic of bNAb is that they recognize epitopes that play a major role in the viral fitness. Quite often they are conformational and in many cases depending on glycosylation and masked by the three dimensional structure of the envelope glycoprotein, only being accessible to the immune system after conformational changes of the envelope glycoprotein. Among the susceptible epitopes to be targeted by bNAbs, the CD4 binding site of gp120 is a valuable region since it plays a major role in HIV-1 infection, it is highly conserved and remains exposed to its ligand. Actually, some of the most powerful broadly neutralizing antibodies recognize this domain in gp120 (IgGb12, VRC01, PGV04 and others). The CD4bs of gp120
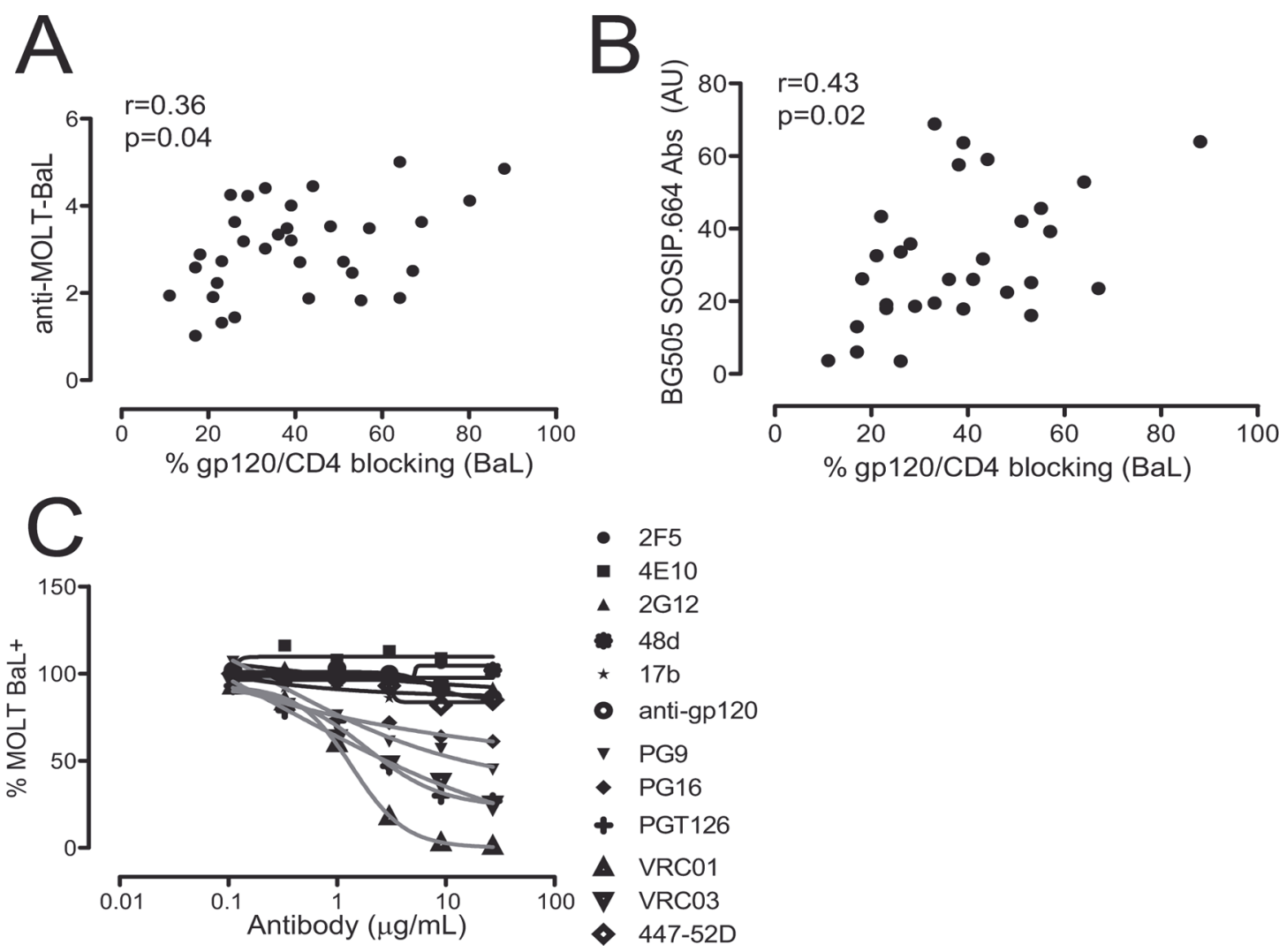

Fig 5. Specificity of gp120/CD4 blocking antibodies. A) The IgG response against Env was evaluated by flow cytometry using MOLT-BaL and uninfectedMOLT cell line. The levels of anti-Env IgG antibodies were calculated as MFI ratio (MOLT-BaL/MOLT). B) Titer of antibodies against the soluble trimer BG505 SOSIP.664 gp140 was determined by ELISA. A correlation analysis between the anti-Env levels and gp120/CD4 blocking activity is shown (Spearman's correlation). C) A set of anti-Env antibodies, whose reactivity is well-known, was used to evaluate the specificity of the antibodies that were able to block the binding of the huCD4mlgG recombinant protein to MOLT-BaL cells. The antibodies tested included CD4bs Abs (VRC01, VRC03), anti-glycosylated epitopes (2G12, PGT126), anti-glycosylated-quaternary epitopes (PG9, PG16), CD4 induced epitopes (17b,48d), anti-gp41 (2F5, 4E10) and a polyclonal goat antigp120 (anti-gp120) obtained by immunization using rgp120.

doi:10.1371/journal.pone.0120648.g005 
is a conformational domain, which comprises a set of amino acids from the $\mathrm{C} 2, \mathrm{C} 3, \mathrm{C} 4$, V5 and C5 regions of gp120 [30]. HIV-1 infected patients develop CD4bs antibodies quite often; however, not all of them are broadly neutralizing indicating that the fine specificity of the antibody and/or the affinity for the ligand may be important to confer neutralizing properties to the antibody molecule [17]. In this way, the main difference between neutralizing and nonneutralizing CD4bs antibodies seems to be the capacity of the former to recognize the CD4bs in its trimeric-native conformation with high affinity [22,31]. Nonneutralizing CD4bs antibodies are able to block the interaction between CD4 and monomeric gp120 but are ineffective at blocking the binding to the native envelope glycoprotein since they are not able to bind to the trimeric protein [22], probably due to the conformational masking of the receptor binding domain of gp120 [32].

To evaluate the frequency of neutralizing CD4bs antibodies in HIV-1 infected individuals we have compared binding to core proteins exposing the CD4bs, binding to soluble and membrane expressed trimeric Env glycoproteins and functional ability to block CD4 binding in plasma samples from HIV-1 infected patients. We have developed a competitive flow cytometry-based functional assay that enables the identification of gp120/CD4 blocking antibodies in the context of a native envelope glycoprotein expressed on the surface of HIV-1-infected cells. This assay is based on the capacity of plasma samples to block the binding of a recombinant huCD4mIgG fusion protein to Env proteins expressed on the surface of infected cells. Using this assay we have been able to determine that the presence of gp120/CD4 blocking antibodies is very common among untreated HIV-1 chronically-infected patients. About $97 \%$ of patients showed gp120/CD4 blocking antibodies and this functional activity correlated with the recognition of the soluble and membrane expressed native glycoprotein, even though both came from different HIV-1 strain (clade A and B). Although neutralizing CD4bs antibodies are able to carry out this blocking activity, the titer of CD4bs antibodies and the gp120/CD4 blocking activity did not show significant correlation. This lack of association indicated that not all CD4bs antibodies can efficiently inhibit the interaction between gp 120 and CD4. Actually, some patients with high titer of CD4bs antibodies showed poor gp120/CD4 inhibition activity indicating that these antibodies might be nonneutralizing antibodies. Conversely, some plasma samples showing undetectable levels of CD4bs antibodies were able to block the interaction between gp120 and its receptor, indicating that CD4bs antibodies that are able to recognize both RSC3 and RSC3 $\Delta$ proteins or antibodies elicited against other epitopes may also show blocking capacity. Actually, most of these plasma samples showed IgGs that were able to bind to trimeric Env glycoprotein. In this line, the PGT126, PG9 and PG16 bNAbs, despite not recognizing the CD4 binding site, showed a certain degree of gp 120/CD4 blocking activity, probably in an allosteric way. A similar mechanism has been also postulated for the PGT121 antibody [33]. Whereas antibodies such as $2 \mathrm{G} 12$ or anti-V3, which recognize other epitopes than the CD4bs in gp120, blocked the virus binding to target cells [34,35]; they failed to block CD4mIgG binding to trimeric Env. In addition, other antibodies, such as 2F5 and 4E10 (anti-MPER) or 48d and 17b (CD4 induced epitopes), were also unable to block the interaction between huCD4mIgG and gp120. A similar lack of inhibition was observed using a goat anti-gp120 polyclonal antibody generated by immunization with recombinant gp120. These data and the correlation observed with total anti-Env antibodies strongly suggest that the gp120/CD4 blocking activity detected in plasma samples from ART-naïve HIV-1 infected patients are the result of definite specificities rather than a polyclonal humoral response against Env. Interestingly, gp120/CD4 inhibition in our assay correlated with the neutralizing capacity of plasma samples measured as reciprocal IC50 of neutralization for both NL4.3 and BaL isolates but not for the primary isolates AC10 and SVBP16. Altogether, the results indicated that these antibodies may contribute to neutralizing capacity of plasma but an important fraction of them are not cross-reactive. 
Although the titer of these antibodies, measured as the percentage of gp120/CD4 inhibition, showed a poor correlation with viral load, patients with a viral load over 5000 copies $/ \mathrm{mL}$ showed the highest titers (data not shown), suggesting that certain amount of antigen should be needed for generating this sort of antibodies and/or that the virus of these patients are resistant to the action of the autologous neutralizing antibodies as it has been previously shown $[36,37]$. In addition, a positive correlation with the time after diagnosis was observed and when a one-year after samples were analyzed, they showed an increase titer of these antibodies supporting the idea that gp120/CD4 blocking antibodies, like neutralizing antibodies, increase over the course of infection.

It has been shown that HIV-1 infection induces a drastic alteration in the biology of B cells, affecting mainly to the memory subset. The loss of these cells correlates with the lack of protective antibodies against vaccines and recall pathogens in HIV-1 infected patients [38]. Interestingly, in our study the titer of these antibodies seem to be stable at least during one year, strongly suggesting that memory humoral response is preserved in these patients, at least with regard to this specificity. To determine the specificity of these antibodies, which cells are responsible for their production and whether viremia could contribute to maintain them are important questions that need further studies.

\section{Conclusions}

The present study shows that almost all HIV-1 infected patients can develop antibodies that are able to block the binding of gp120 to its receptor. These antibodies include not only those elicited against the $\mathrm{CD} 4 \mathrm{bs}$ but also those recognizing a limited number of other unrelated envelope epitopes. These latter antibodies may play a key role in the neutralizing capacity of plasma although, in some cases, with modest amplitude. To improve our knowledge about this sort of antibodies would be greatly helpful for the design of new immunogens which would be able to drive the maturation of these antibodies towards the acquisition of broadly neutralizing abilities.

\section{Supporting Information}

S1 Fig. MOLT T cells chronically infected with HIV-1 express trimeric native Env glycoproteins. MOLT-NL4.3 cells were stained with antibodies targeting several epitopes within Env glycoprotein. Despite some antibodies such as CD4bs Abs, 2G12 or PGT126 (A and B) are able to recognize trimeric and monomeric but properly glycosylated protein, respectively; the PG9 and PG16 antibodies recognize only native trimeric Env protein (C).The staining obtained using all of them are comparable, pointing out that the structure of the Env protein on the surface of chronically infected MOLT cells is mainly native. This statement is supported by the negligible signal obtained using antibodies that recognize epitopes exposed only after gp120/ CD4 interaction such as $17 \mathrm{~b}$ and $48 \mathrm{~d}(\mathrm{D})$, and the low signal achieved using antibodies elicited against poor expose epitopes such as anti-MPER antibodies (2F5)(E). (TIF)

S2 Fig. Plasma samples from HIV-1 infected individuals contain antibodies that bind to trimeric Env glycoproteins. A) IgG response against Env-trimeric glycoprotein was evaluated by ELISA, using the newly generated soluble trimer BG505 SOSIP.664 gp140, and B) by flow cytometry, using the MOLT-BaL and uninfected MOLT cells. Specific signal was calculated as the ratio between the MFI obtained with both cell lines. Dotted line shows the positive cut-off that was calculated as mean $+2 \mathrm{xSD}$ of the values obtained with plasma samples from uninfected healthy controls. Mann-Whitney test was applied. C) Spearman's correlation analysis between 
the antibody titers calculated in A and B showed a weak but positive correlation between both parameters. Note the BG505 SOSIP.664 is subtype A and BaL is subtype B.

(TIF)

\section{Acknowledgments}

We would like to thank to Dan Ouchi for his help with data representation and statistical analysis. The following reagents were obtained through the NIH AIDS Reagent Program, Division of AIDS, NIAID, NIH: VRC01, VRC03, PG9, PG16, PGT126, RSC3 and RSC3 3711 .

\section{Author Contributions}

Conceived and designed the experiments: JC JB BC. Performed the experiments: JC LMM MLR SM EG. Analyzed the data: JC LMM MLR SM EG. Contributed reagents/materials/analysis tools: RWS RD. Wrote the paper: JC JB LMM.

\section{References}

1. Tomaras GD, Yates NL, Liu P, Qin L, Fouda GG, Chavez LL, et al. Initial B-cell responses to transmitted human immunodeficiency virus type 1: virion-binding immunoglobulin $\mathrm{M}(\operatorname{lgM})$ and $\lg \mathrm{G}$ antibodies followed by plasma anti-gp41 antibodies with ineffective control of initial viremia. J Virol. 2008; 82: 1244963. doi: 10.1128/JVI.01708-08 PMID: 18842730

2. Mascola JR, Montefiori DC. The role of antibodies in HIV vaccines. Annu Rev Immunol. 2010; 28: 41344. doi: 10.1146/annurev-immunol-030409-101256 PMID: 20192810

3. West AP, Scharf L, Scheid JF, Klein F, Bjorkman PJ, Nussenzweig MC. Structural insights on the role of antibodies in HIV-1 vaccine and therapy. Cell. 2014; 156: 633-48. doi: 10.1016/j.cell.2014.01.052 PMID: 24529371

4. Kwong PD, Mascola JR, Nabel GJ. Broadly neutralizing antibodies and the search for an HIV-1 vaccine: the end of the beginning. Nat Rev Immunol. 2013; 13: 693-701. doi: 10.1038/nri3516 PMID: 23969737

5. Barbas CF 3rd, Bjorling E, Chiodi F, Dunlop N, Cababa D, Jones TM, et al. Recombinant human Fab fragments neutralize human type 1 immunodeficiency virus in vitro. Proc Natl Acad Sci U S A. 1992; 89 : 9339-9343. PMID: 1384050

6. Wu X, Yang Z-Y, Li Y, Hogerkorp C-M, Schief WR, Seaman MS, et al. Rational design of envelope identifies broadly neutralizing human monoclonal antibodies to HIV-1. Science. 2010; 329: 856-861. doi: 10.1126/science.1187659 PMID: 20616233

7. Moulard M, Phogat SK, Shu Y, Labrijn AF, Xiao X, Binley JM, et al. Broadly cross-reactive HIV-1-neutralizing human monoclonal Fab selected for binding to gp120-CD4-CCR5 complexes. Proc Natl Acad Sci U S A. 2002; 99: 6913-8. PMID: 11997472

8. Muster T, Steindl F, Purtscher M, Trkola A, Klima A, Himmler G, et al. A conserved neutralizing epitope on gp41 of human immunodeficiency virus type 1. J Virol. 1993; 67: 6642-6647. PMID: 7692082

9. Zwick MB, Labrijn a F, Wang M, Spenlehauer C, Saphire EO, Binley JM, et al. Broadly neutralizing antibodies targeted to the membrane-proximal external region of human immunodeficiency virus type $1 \mathrm{gly}$ coprotein gp41. J Virol. 2001; 75: 10892-905. PMID: 11602729

10. Huang J, Ofek G, Laub L, Louder MK, Doria-Rose NA, Longo NS, et al. Broad and potent neutralization of HIV-1 by a gp41-specific human antibody. Nature.2012; 491: 406-412. doi: 10.1038/nature11544 PMID: 23151583

11. Scanlan CN, Pantophlet R, Wormald MR, Ollmann Saphire E, Stanfield R, Wilson I a., et al. The Broadly Neutralizing Anti-Human Immunodeficiency Virus Type 1 Antibody 2G12 Recognizes a Cluster of 1>2 Mannose Residues on the Outer Face of gp120. J Virol. 2002; 76: 7306-7321. PMID: 12072529

12. Walker LM, Phogat SK, Chan-Hui P-Y, Wagner D, Phung P, Goss JL, et al. Broad and potent neutralizing antibodies from an African donor reveal a new HIV-1 vaccine target. Science. 2009; 326: 285-9. doi: 10.1126/science.1178746 PMID: 19729618

13. Pietzsch J, Scheid JF, Mouquet H, Klein F, Seaman MS, Jankovic M, et al. Human anti-HIV-neutralizing antibodies frequently target a conserved epitope essential for viral fitness. J Exp Med. 2010; 207: 1995-2002. doi: 10.1084/jem.20101176 PMID: 20679402 
14. Veazey RS, Shattock RJ, Pope M, Kirijan JC, Jones J, Hu Q, et al. Prevention of virus transmission to macaque monkeys by a vaginally applied monoclonal antibody to HIV-1 gp120. Nat Med.2003; 9: 343346. PMID: 12579198

15. Hessell AJ, Hangartner L, Hunter M, Havenith CEG, Beurskens FJ, Bakker JM, et al. Fc receptor but not complement binding is important in antibody protection against HIV. Nature. 2007; 449: 101-4. PMID: 17805298

16. Li Y, Migueles SA, Welcher B, Svehla K, Phogat A, Louder MK, et al. Broad HIV-1 neutralization mediated by CD4-binding site antibodies. Nat Med.2007; 13: 1032-1034. PMID: 17721546

17. Lynch RM, Tran L, Louder MK, Schmidt SD, Cohen M, DerSimonian R, et al. The Development of CD4 Binding Site Antibodies during HIV-1 Infection. J Virol. 2012; 86: 7588-7595. doi: 10.1128/JVI.0073412 PMID: 22573869

18. Sanchez-Palomino S, Massanella M, Carrillo J, Garcia A, Garcia F, Gonzalez N, et al. A cell-to-cell HIV transfer assay identifies humoral responses with broad neutralization activity. Vaccine.2011; 29: 52505259. doi: 10.1016/j.vaccine.2011.05.016 PMID: 21609746

19. Blanco J, Barretina J, Clotet B, Este JA. R5 HIV gp120-mediated cellular contacts induce the death of single CCR5-expressing CD4 T cells by a gp41-dependent mechanism. J Leukoc Biol. 2004; 76: 804811. PMID: 15258189

20. Walker LM, Huber M, Doores KJ, Falkowska E, Pejchal R, Julien JP, et al. Broad neutralization coverage of HIV by multiple highly potent antibodies. Nature.2011; 477: 466-470. doi: 10.1038/nature10373 PMID: 21849977

21. Carrillo J, Restrepo C, Rallon NI, Massanella M, del Romero J, Rodriguez C, et al. HIV exposed seronegative individuals show antibodies specifically recognizing native HIV envelope glycoprotein. AIDS.2013; 27: 1375-1385. doi: 10.1097/QAD.0b013e32835fac08 PMID: 23945502

22. Sanders RW, Derking R, Cupo A, Julien JP, Yasmeen A, de Val N, et al. A next-generation cleaved, soluble HIV-1 Env Trimer, BG505 SOSIP.664 gp140, expresses multiple epitopes for broadly neutralizing but not non-neutralizing antibodies. PLoS Pathog.2013; 9: e1003618. doi: 10.1371/journal.ppat. 1003618 PMID: 24068931

23. Montefiori DC. Evaluating neutralizing antibodies against HIV, SIV, and SHIV in luciferase reporter gene assays. Curr Protoc Immunol. 2005;Chapter 12: Unit 12 11. doi: 10.1002/0471142735. im1211s64

24. Hessell AJ, Poignard P, Hunter M, Hangartner L, Tehrani DM, Bleeker WK, et al. Effective, low-titer antibody protection against low-dose repeated mucosal SHIV challenge in macaques. Nat Med. 2009; 15: 951-954. doi: 10.1038/nm.1974 PMID: 19525965

25. Baba TW, Liska V, Hofmann-Lehmann R, Vlasak J, Xu W, Ayehunie S, et al. Human neutralizing monoclonal antibodies of the IgG1 subtype protect against mucosal simian-human immunodeficiency virus infection. Nat Med.2000; 6: 200-206. PMID: 10655110

26. Hessell AJ, Rakasz EG, Poignard P, Hangartner L, Landucci G, Forthal DN, et al. Broadly neutralizing human anti-HIV antibody 2 G12 is effective in protection against mucosal SHIV challenge even at low serum neutralizing titers. PLoS Pathog.2009; 5: e1000433. doi: 10.1371/journal.ppat.1000433 PMID: 19436712

27. Mascola JR, Stiegler G, VanCott TC, Katinger H, Carpenter CB, Hanson CE, et al. Protection of macaques against vaginal transmission of a pathogenic HIV-1/SIV chimeric virus by passive infusion of neutralizing antibodies. Nat Med.2000; 6: 207-210. PMID: 10655111

28. Shibata R, Igarashi T, Haigwood N, Buckler-White A, Ogert R, Ross W, et al. Neutralizing antibody directed against the HIV-1 envelope glycoprotein can completely block HIV-1/SIV chimeric virus infections of macaque monkeys. Nat Med.1999; 5: 204-210. PMID: 9930869

29. Huang KH, Bonsall D, Katzourakis A, Thomson EC, Fidler SJ, Main J, et al. B-cell depletion reveals a role for antibodies in the control of chronic HIV-1 infection. Nat Commun. 2010; 1:102. doi: 10.1038/ ncomms1100 PMID: 20981030

30. Kwong PD, Wyatt R, Robinson J, Sweet RW, Sodroski J, Hendrickson WA. Structure of an HIV gp120 envelope glycoprotein in complex with the CD4 receptor and a neutralizing human antibody. Nature. 1998; 393: 648-659. PMID: 9641677

31. Pantophlet R, Ollmann Saphire E, Poignard P, Parren PW, Wilson IA, Burton DR. Fine mapping of the interaction of neutralizing and nonneutralizing monoclonal antibodies with the CD4 binding site of human immunodeficiency virus type $1 \mathrm{gp} 120$. J Virol.2003; 77: 642-658. PMID: 12477867

32. Kwong PD, Doyle ML, Casper DJ, Cicala C, Leavitt SA, Majeed S, et al. HIV-1 evades antibody-mediated neutralization through conformational masking of receptor-binding sites. Nature.2002; 420: 678682. PMID: 12478295 
33. Julien JP, Sok D, Khayat R, Lee JH, Doores KJ, Walker LM, et al. Broadly neutralizing antibody PGT121 allosterically modulates CD4 binding via recognition of the HIV-1 gp120 V3 base and multiple surrounding glycans. PLoS Pathog.2013; 9: e1003342. doi: 10.1371/journal.ppat.1003342 PMID: 23658524

34. Valenzuela A, Blanco J, Krust B, Franco R, Hovanessian AG. Neutralizing antibodies against the V3 loop of human immunodeficiency virus type $1 \mathrm{gp} 120$ block the CD4-dependent and-independent binding of virus to cells. J Virol.1997; 71: 8289-8298. PMID: 9343181

35. Ugolini S, Mondor I, Parren PW, Burton DR, Tilley SA, Klasse PJ, et al. Inhibition of virus attachment to CD4+ target cells is a major mechanism of T cell line-adapted HIV-1 neutralization.1997; 186: 12871298. PMID: 9334368

36. Wei X, Decker JM, Wang S, Hui H, Kappes JC, Wu X, et al. Antibody neutralization and escape by HIV1. Nature.2003; 422: 307-312. PMID: 12646921

37. Richman DD, Wrin T, Little SJ, Petropoulos CJ. Rapid evolution of the neutralizing antibody response to HIV type 1 infection. Proc Natl Acad Sci U S A.2003; 100: 4144-4149. PMID: 12644702

38. Titanji K, De Milito A, Cagigi A, Thorstensson R, Grutzmeier S, Atlas A, et al. Loss of memory B cells impairs maintenance of long-term serologic memory during HIV-1 infection. Blood.2006; 108: 1580-1587. PMID: 16645169 